\title{
Elastic electron scattering from water vapor and ice at high momentum transfer
}

\author{
M. Vos, ${ }^{1}$ E. Weigold,${ }^{1}$ and R. Moreh ${ }^{2}$ \\ ${ }^{1}$ Atomic and Molecular Physics Laboratories, Research School of Physics and Engineering, \\ Australian National University, Canberra, ACT 0200, Australia \\ ${ }^{2}$ Department of Physics, Ben-Gurion University of the Negev, Beer-Sheva 84105, Israel
}

(Received 19 November 2012; accepted 30 December 2012; published online 24 January 2013)

\begin{abstract}
We compare the area, peak separation, and width of the $\mathrm{H}$ and $\mathrm{O}$ elastic peak for light and heavy water, as observed in spectra of keV electrons scattered over large angles. Peak separation is well reproduced by the theory, but the $\mathrm{O}: \mathrm{H}$ area ratio is somewhat larger than expected and is equal to the O:D area ratio. Thus no anomalous scattering from $\mathrm{H}$ was observed. Only minor differences are observed for scattering from a gaseous or a solid target. The extracted mean kinetic energy of H and D agreed within 5\% with the calculated ones for ice. For the more difficult vapor measurements agreement was on a $12 \%$ level. A preliminary attempt to extract the O kinetic energy in ice agreed within $10 \%$ with the calculated values. (C) 2013 American Institute of Physics. [http://dx.doi.org/10.1063/1.4775810]
\end{abstract}

\section{INTRODUCTION}

Elastic electron scattering from atoms and molecules at high momentum transfer is a new technique for the direct observation of the momentum distribution and the kinetic energies of the scattering nuclei. An electron scattering "quasielastically" from water at high momentum transfer, interacts not with the molecule as a whole but with a single nucleus (either $\mathrm{H}$ or $\mathrm{O}$ ). The electron energy changes (due to the recoil) as if it was scattering from free $\mathrm{H}$ or $\mathrm{O}$ particles. As the recoil energy depends on the mass of the scattering atom, two separate "elastic" peaks are observed. The width of the peaks is interpreted as a Doppler profile of the nuclear motion of the $\mathrm{H}$ and $\mathrm{O}$ atoms. In recent years $\mathrm{e}^{-}$-scattering was used to study the kinetic energy of $\mathrm{H}$ in methane $\mathrm{e}^{1,2}$ and the value obtained was very close to the calculated values. The purpose of studying the electron scattering from $\mathrm{H}_{2} \mathrm{O}$ and $\mathrm{D}_{2} \mathrm{O}$ is twofold. First, to measure the kinetic energies of the H-, D-, and $\mathrm{O}$-nuclei in $\mathrm{H}_{2} \mathrm{O}$ and $\mathrm{D}_{2} \mathrm{O}$ to find out to what extent the measured values can be theoretically reproduced. Second, to search for an anomaly in the electron scattering cross section of hydrogen in $\mathrm{H}_{2} \mathrm{O}$ as compared to that of $\mathrm{D}$ in $\mathrm{D}_{2} \mathrm{O}$.

Direct observation of the momentum distribution of atoms in molecules and solids is restricted to very few techniques. The main technique for such studies has been neutron Compton scattering (NCS), ${ }^{3}$ but other techniques, based on resonance photon scattering, have also been used to measure the mean kinetic energy of the scattering atoms. ${ }^{4-6}$ In recent years it has become evident that, when done at high energies (more than $1 \mathrm{keV}$ ), in a backscattering geometry, and with sufficient energy resolution (better than $1 \mathrm{eV}$ ), elastic electron scattering probes the atomic momentum distribution in a very similar way to $\mathrm{NCS}^{7-9}$ In spite of the fact that these electron scattering experiments are relatively simple "table-top" laboratory experiments, there is relatively little work published on this technique, and experimentally and theoretically this technique is still in its infancy. Here we explore the possibilities of this technique for the case of water. It is studied both as ice and as vapor. Water has been studied extensively with NCS, with recently a lot of emphasis on establishing experimentally the influence of the hydrogen bond on the proton momentum distribution and the kinetic energy in supercooled water. ${ }^{10-12}$ In this study we will limit ourselves to obtaining an estimate of the mean kinetic energy of $\mathrm{H}$ (D) and $\mathrm{O}$ in both $\mathrm{H}_{2} \mathrm{O}$ and $\mathrm{D}_{2} \mathrm{O}$. The only previously published high-momentum transfer study of electrons scattering from ice ${ }^{13}$ resolved the $\mathrm{H}$ contribution only as a shoulder from the main $\mathrm{O}$ elastic peak, and establishing meaningful width, area and $\mathrm{O}-\mathrm{H}$ peak separation was not possible. The present measurements, with better energy resolution, and extending to higher momentum transfers are thus the first electron-based measurement of the mean kinetic energy of $\mathrm{H}, \mathrm{D}$, and $\mathrm{O}$ in water.

Water has also attracted considerable interest due to the "anomalous" intensity ratio of the $\mathrm{H}$ and D signal in neutron scattering measurements of mixtures of heavy and light water. ${ }^{14}$ In that work it was found that the D:H cross section ratio for neutron scattering depends on their relative concentration. However, neutron transmission measurements ${ }^{15}$ failed to find any deviation from the well-known n-p scattering cross section. In addition, similar scattering measurements using neutrons of around the same energy range as Ref. 14 were reported ${ }^{16}$ as well as measurements at much higher energies. ${ }^{17,18}$ Neither did reveal any deficit in the n-p scattering cross section. Later anomalous $\mathrm{C}: \mathrm{H}$ intensity ratios were reported for scattering from polymers (e.g., Refs. 8 and 19) for both neutron and electron scattering. No anomalous intensity ratio was seen for electrons scattered from methane in the gas phase. ${ }^{1}$ This cast doubt on the electron scattering data from polymers, as electron beams are known to deplete hydrogen from a target (radiation damage), whereas the gasphase target was continuously replenished. It is thus of great interest to investigate if $\mathrm{H}$ (or D) shows anomalous intensity, relative to the $\mathrm{O}$ intensity, in either ice or vapor.

In our scattering experiment an electron with known en$\operatorname{ergy}\left(E_{0}\right)$ and momentum $\left(\boldsymbol{k}_{0}\right)$ scatters over an angle $\theta\left(135^{\circ}\right)$ 
and is detected with energy $E_{1}$ and momentum $\boldsymbol{k}_{1}$. For the interpretation of the electron scattering data we rely on the first Born approximation. In this approximation the cross section factors out as

$$
\frac{d^{2} \sigma}{d \Omega d \varepsilon}=\frac{m^{2}}{4 \pi^{2} \hbar^{5}} \frac{k_{1}}{k_{0}} W(\boldsymbol{q}) S(\boldsymbol{q}, \omega),
$$

where $m$ is the mass of the scattering electron and $S(\boldsymbol{q}, \omega)$ is the dynamical structure factor, depending on the momentum transfer $\left(\boldsymbol{q}=\boldsymbol{k}_{1}-\boldsymbol{k}_{0}\right)$ and energy loss $\left(\omega=E_{0}-E_{1}\right) . W(\boldsymbol{q})$ is the square Fourier transform of the electron-target interaction $V(\boldsymbol{r})$, and is a constant for each atom contributing to the spectrum. Thus the shape of the energy loss spectrum is directly proportional to the dynamic structure factor, a target property. Therefore, assuming that the first Born approximation describes these measurements well, we obtain a direct measurement of the shape of $S(\boldsymbol{q}, \omega)$. As stated already in the original work of van Hove, ${ }^{20}$ the nature of the scattering particle, or the details of the projectile-target interaction are largely irrelevant for the separation of the cross section in two factors $S(\boldsymbol{q}, \omega)$ and $W(\boldsymbol{q})$ within the first Born approximation.

The validity of the first Born approximation for electron scattering depends on the value of $E_{0}$. For low values of $E_{0}$ this approximation is very poor. At high enough energies the first Born approximation should work. So far all evidence indicates that, in experiments where $E_{0}$ is high enough to resolve atoms with different mass due to the recoil effect, the spectra are indeed proportional to $S(\boldsymbol{q}, \omega)$. An experimental verification of the validity of the first Born approximation is the good agreement the shape of the spectra obtained with neutron and electron scattering. ${ }^{8,19}$

In the electron scattering case the comparison of the experiment with theory is particularly simple. As $E_{1}$ is only slightly smaller than $E_{0}(0.2 \%$ for scattering from $\mathrm{H}$ over $135^{\circ}$, and smaller differences for scattering from heavier elements), we have to very good approximation $\left|k_{1}\right|=\left|k_{0}\right|$ and as a consequence $|q|=2\left|k_{0}\right| \sin (\theta / 2)$. Thus each spectrum corresponds to a measurement of $S(\boldsymbol{q}, \omega)$ at constant $\boldsymbol{q}$, in contrast to NCS data (in particular for scattering from $\mathrm{H}$ ) where the energy reduction of the neutron in the collision is substantial, and the momentum transfer $\boldsymbol{q}$ varies with the energy transfer in the collision.

In the high momentum-transfer limit, where the plane wave impulse approximation applies, the interpretation of $S(\boldsymbol{q}, \omega)$ for a target, consisting of several elements, becomes very simple. ${ }^{21}$ The contribution of electrons scattering from atom $i$ is then peaked at $\overline{E_{\text {rec }}^{i}}=q^{2} / 2 M_{i}$ with $M_{i}$ the mass of the scattering atom. The width of each peak is Doppler broadened i.e. the width of component $i$ is proportional to the component of the momentum $\boldsymbol{p}_{i}$ of the scattering atom along $q$ :

$$
E_{r e c}^{i}=\frac{q^{2}}{2 M_{i}}+\frac{\mathbf{q} \cdot \mathbf{p}_{\mathbf{i}}}{M_{i}} .
$$

The spectrum is then exactly what would be expected for an electron scattering from free $\mathrm{O}$ and $\mathrm{H}(\mathrm{D})$ particles. In this limit we can obtain the kinetic energy of the scattering particle. If the molecules are randomly oriented in space then the mean kinetic energy of scattering atom $i$ is related to the
TABLE I. The differential cross section as calculated using the ELSEPA package for atomic $\mathrm{H}$ and atomic $\mathrm{O}$ for the energy as indicated and a scattering angle of $135^{\circ}$. With increasing energy the ratio of these cross section approaches $Z_{\mathrm{O}}^{2} / Z_{\mathrm{H}}^{2}=64$, as predicted by the Rutherford formula.

\begin{tabular}{lccc}
\hline $\begin{array}{l}\text { Energy } \\
(\mathrm{keV})\end{array}$ & $\begin{array}{c}\mathrm{dcs} \mathrm{O} \\
\left(\mathrm{cm}^{2} / \mathrm{sr}\right)\end{array}$ & $\begin{array}{c}\mathrm{dcs} \mathrm{H} \\
\left(\mathrm{cm}^{2} / \mathrm{sr}\right)\end{array}$ & $\begin{array}{c}\text { Ratio } \\
\mathrm{dcs} \text { O:dcs H }\end{array}$ \\
\hline 1.5 & $5.74 \times 10^{-20}$ & $7.66 \times 10^{-22}$ & 74.9 \\
2 & $3.15 \times 10^{-20}$ & $4.33 \times 10^{-22}$ & 72.7 \\
2.5 & $1.98 \times 10^{-20}$ & $2.78 \times 10^{-22}$ & 71.1 \\
3 & $1.35 \times 10^{-20}$ & $1.94 \times 10^{-22}$ & 69.9 \\
5 & $4.69 \times 10^{-21}$ & $6.99 \times 10^{-23}$ & 67.2 \\
6 & $3.23 \times 10^{-21}$ & $4.86 \times 10^{-23}$ & 66.6 \\
\hline \hline
\end{tabular}

(energy-resolution corrected) width of peak $i$ (standard deviation $\sigma_{i}$ ) by

$$
\sigma_{i}=\sqrt{\frac{4 \overline{3} \overline{E_{\text {rec }}^{i}} \overline{E_{\text {kin }}^{i}}}{.}}
$$

In order to calculate the intensity ratio of the scattered electrons from either $\mathrm{H}$ or $\mathrm{O}$ we have to know the cross section for scattering of an electron with energy $E_{0}$ over an angle $\theta$ from both atoms. This is not just the Rutherford cross section (which corresponds to scattering from a bare nucleus) as the incoming electron interacts with an electric field that is due to the nucleus and the surrounding electrons. We used the ELSEPA package to calculate the differential cross section for electrons scattering from atomic $\mathrm{H}$ or $\mathrm{O} .{ }^{22}$ This is an atomic calculation and thus neglects the difference between the electron distribution in an atom by itself and in an atom that is part of a molecule. Large-angle deflections, as studied here, correspond classically to small impact parameter collisions, and we expect that in this case the scattering is governed by the potential close to the nucleus. This should not be greatly affected by the redistribution of the electron charge due to the molecular bonds. The differential cross section for $\mathrm{H}$ and $\mathrm{D}$ are identical within the theoretical framework on which ELSEPA is based. The obtained cross sections are reproduced in Table I.

\section{EXPERIMENTAL DETAILS}

The spectrometer used was basically as described in Ref. 2. Differential pumping was added between the electron gun and the main chamber to avoid poisoning by water of the $\mathrm{BaO}$ cathode used in the electron gun. As a consequence the pressure increase, due to the admitted water vapor at the electron gun, was $\simeq 100 \times$ less than the pressure increase in the main chamber.

For the vapor measurements the water molecules effused from a $0.5 \mathrm{~mm}$ diameter needle pointing at the interaction region perpendicular to the plane containing the gun and the detector. The flow rate is controlled by a slightly heated leak valve (temperature $40^{\circ} \mathrm{C}$ ) followed by a $20 \mathrm{~cm}$ tube connected to the needle at the interaction region. Fully opening the leak valve caused a pressure to increase 20 times more than the actual measurement pressure. Thus the main expansion occurs in the leak valve and the gas temperature is expected to equilibrate again to room temperature in the 
tube. The driving pressure behind the needle is thus at most 1 torr $(\approx 1 / 20$ of the water vapor pressure at room temperature: 20 Torr), and we do not expect a dramatic decrease of the temperature while expanding in the needle at this driving pressure. The angular distribution of the translational motion of the emerging molecules will deviate strongly from random, but the translational contribution to the $\mathrm{H}$ width is very small, as the velocity of the center-of-mass of the molecule is low.

For the ice measurements a copper cold finger was added with its front surface lining up with the interaction region. The cold finger was cooled by liquid nitrogen. In the ice experiments the needle directed its flux of vapor towards the cold finger front surface. Using a thermocouple we measured a temperature of $-155^{\circ} \mathrm{C}$ near the measurement surface of the cold finger.

The pressure increased in the spectrometer from 2 $\times 10^{-9}$ Torr to $1-2 \times 10^{-6}$ Torr during the vapor measurement and rose by $1-2 \times 10^{-9}$ Torr for the ice measurements. For ice, even at this low pressure, the impingement rate of water molecules at the measurement surface is sufficient for the growth of about 0.1-1 monolayers of ice a second, as this surface is only a few mm away from the needle from which all water molecules emerge. Thus the sample is continuously replenished, decreasing highly the likelihood that radiation damage will affect the outcome of the ice measurements. For the ice experiments the incoming beam was along the surface normal of the cold finger.

There are slight uncertainties in the exact zero position of the energy loss scale. In this paper, unless otherwise stated, the $\mathrm{O}$ peak is aligned with its calculated recoil of $q^{2} / 2 M$. For the gas phase measurements two spectra were obtained: one while water vapor was admitted to the chamber, and one without. The intensity in the second ("background") spectrum was about $10 \%$ of the intensity obtained with water coming out of the needle. The background spectrum resembles the elastic peak and energy loss spectrum of a metal surface, and presumably is caused by electrons making their way into the analyser after scattering from one or more surfaces. The background spectrum, normalised by the charge collected in the Faraday cup, was subtracted from the signal spectrum, as shown in Fig. 1.

The energy scale of the spectrometer was calibrated using a Xe vapor spectrum. The distance from the Xe elastic peak to the first excited state $\left(6 s\left[1 \frac{1}{2}\right]^{\circ}\right)$ was put to the accurately known value of $8.44 \mathrm{eV}^{23}$ Usually the voltages of the spectrometer were adjusted so the energy loss area of interest was scanned over the two-dimensional detector. This averages out the inhomogeneities of the channel plate response, and this operation mode resulted in an energy resolution of $\approx 0.4 \mathrm{eV}$. If maximum resolution was required, the spectrum was measured without scanning, eliminating the broadening due to imperfections in the energy calibration of the spectrometer. The resolution was then estimated to be $\approx 0.3 \mathrm{eV}$.

The spectra were fitted with great care. There are some intrinsic problems in fitting a spectrum where the height of the $\mathrm{H}$ component is $100 \times$ less than the height of the $\mathrm{O}$ peak. Fitting the $\mathrm{O}$ peak with a single Gaussian works at first sight quite well, but if one magnifies the vertical scale such that the $\mathrm{H}$ peak becomes visible, it is clear that a single Gaussian fit

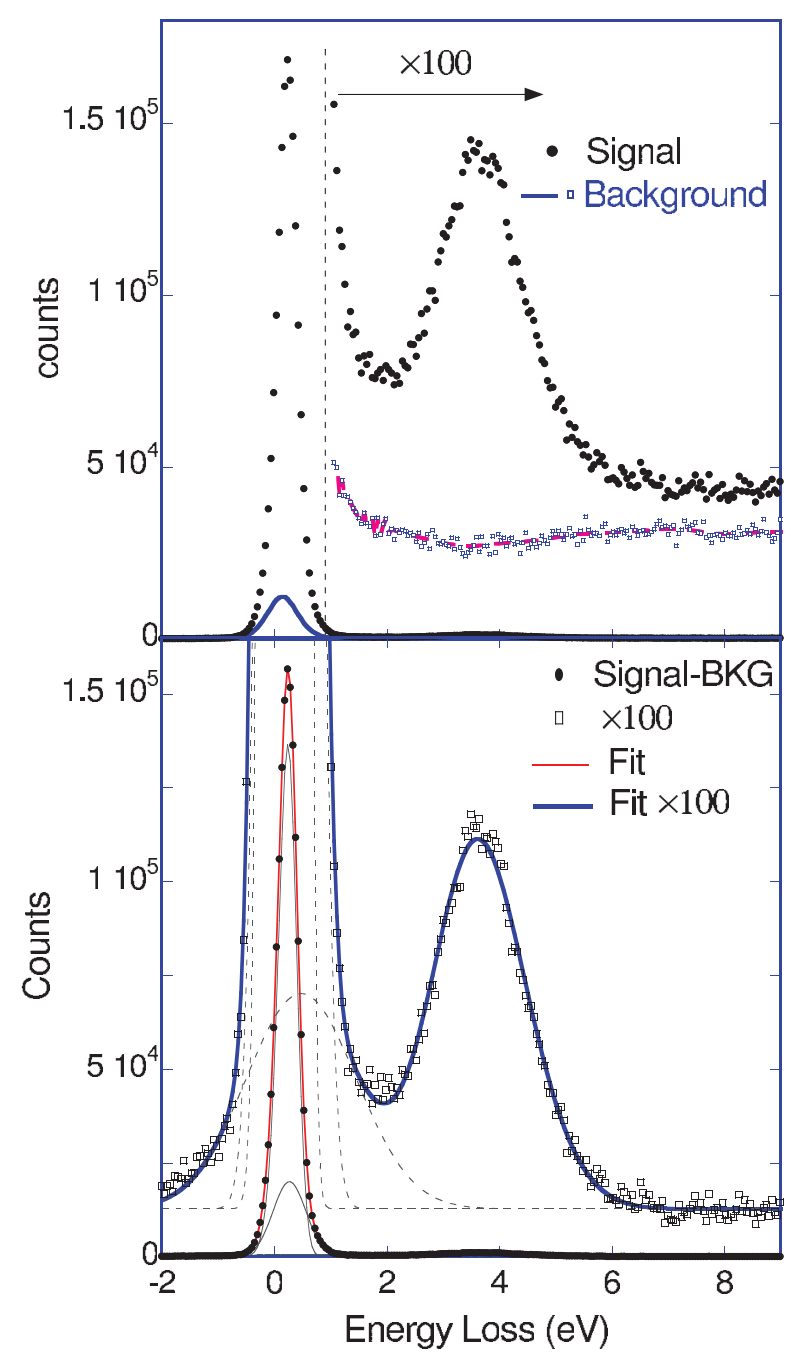

FIG. 1. The top figure shows the background subtraction procedure for the case of $2 \mathrm{keV}$ electrons scattering from water vapor. The background spectrum, obtained with the leak valve closed, was subtracted from the spectrum obtained with the leak valve admitting $\mathrm{H}_{2} \mathrm{O}$ vapor. The lower half gives an example of the fitting procedure after the background subtraction. The height of the $\mathrm{H}$ peak is $\approx 100$ less than the height of the $\mathrm{O}$ peak. The shape of the $\mathrm{O}$ peak cannot be described by a single Gaussian. Here a good fit of this peak was obtained using 3 Gaussians of different width and at slightly different positions (gray lines, gray dashed lines $\times 100$ ). The $\mathrm{H}$ peak is fitted by a single Gaussian.

of the $\mathrm{O}$ peak drops off much too quickly. The fitting program then tries to increase the width of the H component (and constant background) to reduce the deviation near the $\mathrm{O}$ peak. Hence, it was necessary to fit the O peak with 3-4 Gaussian components, each with different width and located at slightly different (up to $0.4 \mathrm{eV}$ ) positions. Only then can we describe the base of the $\mathrm{O}$ peak well, and extract meaningful information on the $\mathrm{H}$ peak from the fit. The fitting procedure is illustrated in Fig. 1 for the case of $2 \mathrm{keV}$ electrons scattering from $\mathrm{H}_{2} \mathrm{O}$ vapor.

\section{CALCULATION OF ATOMIC KINETIC ENERGIES}

The method of calculating the kinetic energy $K_{e}(\mathrm{H})$ of the $\mathrm{H}$-atom in $\mathrm{H}_{2} \mathrm{O}$ was explained in Ref. 24, where a decoupling 
TABLE II. List of the literature experimental vibration and libration frequencies ${ }^{25-30}$ of $\mathrm{H}_{2} \mathrm{O}$ and $\mathrm{D}_{2} \mathrm{O}$ (in $\mathrm{cm}^{-1}$ units) used in the present calculations. In all cases the simulated libration and translation values ${ }^{28,29}$ are listed together with the structure parameters ${ }^{28-30}$ of the vapor and ice phases of $\mathrm{H}_{2} \mathrm{O}$ and $\mathrm{D}_{2} \mathrm{O}$.

\begin{tabular}{lccccc}
\hline \hline & \multicolumn{3}{c}{ Vapor $^{25}$} & & \multicolumn{2}{c}{ Ice $I h^{26-30}$} \\
\cline { 2 - 3 } \cline { 5 - 6 } & $\mathrm{H}_{2} \mathrm{O}$ & $\mathrm{D}_{2} \mathrm{O}$ & & $\mathrm{H}_{2} \mathrm{O}$ & $\mathrm{D}_{2} \mathrm{O}$ \\
\hline Stretch & 3686,3738 & 2666,2789 & & 3085,3220 & 2240,2360 \\
Bend & 1596 & 1179 & & 1650 & 1200 \\
Libration & $\ldots$ & $\ldots$ & & $380-935$ & $274-670$ \\
Translation & $\ldots$ & $\ldots$ & & $20-400$ & $20-380$ \\
OH(D) dist. $(\AA)$ & 0.958 & 0.958 & & 0.985 & 0.985 \\
HOH angle & $104.3^{\circ}$ & $104.3^{\circ}$ & & $108.4^{\circ}$ & $108.4^{\circ}$ \\
\hline \hline
\end{tabular}

between the translational, rotational, and internal vibrational motions was assumed. We used the same procedure for calculating the $K_{e}(\mathrm{D})$ in $\mathrm{D}_{2} \mathrm{O}$. Briefly $\mathrm{K}_{e}(\mathrm{H}, \mathrm{D})$ is contributed by both translation and rotation (external modes) of the entire molecule and also by the internal vibrational modes of the $\mathrm{D}_{2} \mathrm{O}$ molecule. The expression of $K_{e}(\mathrm{D})$ is written as ${ }^{24}$

$$
K_{e}(\mathrm{H}, \mathrm{D})=\mathrm{S}_{\mathrm{t}} \frac{3}{2} \mathrm{kT}+\mathrm{S}_{\mathrm{r}} \frac{3}{2} \mathrm{kT}+\sum_{\mathrm{j}=1}^{3} \mathrm{~S}_{\mathrm{j}} \frac{1}{2}\left(\frac{\mathrm{h} v_{\mathrm{j}}}{\mathrm{e}^{\mathrm{h} \nu_{\mathrm{j}} / \mathrm{kT}}-1}+\frac{\mathrm{h} v_{\mathrm{j}}}{2}\right) .
$$

The first two terms on the right of Eq. (4) represent the classical expression of translational and rotational energy of the entire molecule with $S_{t}$ and $S_{r}$ the fractions shared by $\mathrm{H}(\mathrm{D})$-atom in each mode. In calculating the contribution of the internal vibrational modes (contained in the third term), the harmonic approximation was assumed. The internal vibrations consist of three modes: stretch of the $\mathrm{OH}(\mathrm{OD})$ bonds, bend of the HOH (DOD) angle and HH (DD) stretch. Each mode of vibration is represented by a harmonic oscillator with frequency $v_{j}$ where $S_{j}$ is the kinetic energy fraction shared by the H(D)-atom in each mode. The force constants $k_{1}, k_{2}$, and $k_{3}$ of the above three modes were deduced by using the literature input data of $\mathrm{D}_{2} \mathrm{O}$ given in Table II (for the solid and vapor phases) and using computational methods of infrared spectroscopy. The results enabled us to deduce the kinetic energy fractions $S_{j}$ shared by the D-nucleus in each mode. Both $\mathrm{H}_{2} \mathrm{O}$ and $\mathrm{D}_{2} \mathrm{O}$ have the same force constants. The results are given in Table III. Note that in the vapor phase it was necessary to introduce small changes in the values of the force constants to better reproduce the experimental vibrational fre- quencies of $\mathrm{D}_{2} \mathrm{O}$. It turned out that such differences in the force constants had only minor effects on the $K_{e}(\mathrm{D})$ values in $\mathrm{D}_{2} \mathrm{O}$. Table IV lists the calculated $K_{e}(\mathrm{D})$ and $K_{e}(\mathrm{H})$ and $K_{e}(\mathrm{O})$ values of $\mathrm{H}_{2} \mathrm{O} / \mathrm{D}_{2} \mathrm{O}$.

\section{RESULTS}

\section{A. Growth of ice layer}

An example of an ice growth sequence is shown in Fig. 2, top panel for the case of $6 \mathrm{keV}$ electrons scattered from a growing layer of $\mathrm{D}_{2} \mathrm{O}$ ice. At this high incoming electron energy the recoil effect allows us to monitor the growth of ice as electrons scattered from $\mathrm{Cu}$ and from $\mathrm{O}$ appear at noticeably different energy losses. Before water exposure we obtain from the cold finger a spectrum with a sharp peak at small energy loss followed by a broader distribution at higher energy losses. The sharp peak we assign to $\mathrm{Cu}$ and the tail due to $\mathrm{C}$ and $\mathrm{O}$ impurities, as expected for a surface that has not been cleaned in vacuum. Spectra were collected for short periods (a few minutes) after the start of $\mathrm{D}_{2} \mathrm{O}$ exposure. Soon the $\mathrm{Cu}$ peak is no longer the largest component and after $15 \mathrm{~min}$ or more, the $\mathrm{Cu}$ peak becomes undetectable. The inelastic mean free path for $6 \mathrm{keV}$ electrons in water is $\approx 150 \AA$. Under these conditions (relatively high- $Z$ substrate with a low $Z$ overlayer) we expect to be able to detect $\mathrm{Cu}$ for ice thicknesses of less than 2 times the inelastic mean free path. ${ }^{31}$ Thus we obtain a rough estimate of the ice growth rate of $20 \AA / \mathrm{min}$.

The spectra at larger energy losses change completely as well. These are shown in the lower panel of Fig. 2. Before water exposure we see a slowly increasing, rather feature-less spectrum, as expected for a $\mathrm{Cu}$ surface (without surface cleaning). After a thick ice layer is grown two additional features are seen. One Gaussian-shaped feature appears, and its energy loss depends on the incoming energy. This is due to electrons scattered from $\mathrm{H}$ or $\mathrm{D}$, as the recoil energy increases linearly with the incoming energy. This feature is not seen in ordinary energy loss spectra of water as these are taken at much lower momentum transfer. The second feature is due to electron excitation in ice and its onset is about $8 \mathrm{eV}$ below the $\mathrm{O}$ elastic peak. These electrons have scattered elastically from $\mathrm{O}$, and created an electronic excitation. The shape of this spectrum resembles that seen in electron microscopy measurements of thin layers of ice. ${ }^{32}$

At lower incoming energies, where the technique is more surface sensitive, the transition from a " $\mathrm{Cu}$ " energy loss spectrum to an ice energy loss spectrum is even faster.

TABLE III. The force constants $k_{1}, k_{2}$, and $k_{3}$ of OD stretch, DOD bend and DD stretch (in $10^{5}$ dyn/cm units) that we deduced to yield the best agreement with the literature experimental frequencies of the various phases of $\mathrm{D}_{2} \mathrm{O}$ listed in Table II. The energy fractions $S_{j}$ of the D- and O-atoms in each mode of vibration is also listed where $S_{r}$ and $S_{t}$ are the energy fractions in rotation/libration and translation. A similar table for the case of $\mathrm{H}_{2} \mathrm{O}$ was given in Ref. 24 .

\begin{tabular}{|c|c|c|c|c|c|c|c|c|}
\hline $\begin{array}{l}\mathrm{D}_{2} \mathrm{O} \\
\text { Phases }\end{array}$ & $\begin{array}{c}k_{1} \\
\text { OD str. }\end{array}$ & $\begin{array}{c}k_{2} \\
\text { DOD bend }\end{array}$ & $\begin{array}{c}k_{3} \\
\text { DD str. }\end{array}$ & $S_{1}$ & $S_{2}$ & $S_{3}$ & $S_{r}$ & $S_{t}$ \\
\hline Vapor-D & 7.810 & 0.650 & 0.044 & 0.4465 & 0.4547 & 0.4323 & 0.4561 & 0.1 \\
\hline Vapor-O & & & & 0.1070 & 0.0906 & 0.1354 & 0.0878 & 0.8 \\
\hline Ice Ih-D & 6.020 & 0.578 & 0.482 & 0.4307 & 0.4289 & 0.4689 & 0.4572 & 0.1 \\
\hline Ice Ih-O & & & & 0.1387 & 0.1422 & 0.0622 & 0.0856 & 0.8 \\
\hline
\end{tabular}


TABLE IV. Average measured and calculated $\mathrm{Ke}(\mathrm{D}), \mathrm{Ke}(\mathrm{H})$, and $\mathrm{Ke}(\mathrm{O})$ (in meV units) for vapor $(\mathrm{T}=295 \mathrm{~K})$ and ice $(\mathrm{T}=118 \mathrm{~K})$ phases in both $\mathrm{H}_{2} \mathrm{O}$ and $\mathrm{D}_{2} \mathrm{O}$. The incident electron energies of the measurements were between 1.5 and $6.0 \mathrm{keV}$. The calculated values are obtained using Eq. (4). All input data for the calculations are those of Table II. For ice Ih, optical frequencies were used together with the simulated libration spectra of Refs. 28 and 29 . The vapor results at $295 \mathrm{~K}$ were calculated by assuming free rotations of $\mathrm{H}_{2} \mathrm{O}$ and $\mathrm{D}_{2} \mathrm{O}$.

\begin{tabular}{|c|c|c|c|c|c|c|c|c|c|}
\hline \multirow[b]{3}{*}{ Sample } & \multirow[b]{3}{*}{$\mathrm{T}(\mathrm{K})$} & \multicolumn{4}{|c|}{$\mathrm{H}_{2} \mathrm{O}$} & \multicolumn{4}{|c|}{$\mathrm{D}_{2} \mathrm{O}$} \\
\hline & & \multicolumn{2}{|c|}{$K_{e}(\mathrm{H})(\mathrm{meV})$} & \multicolumn{2}{|c|}{$K_{e}(\mathrm{O})(\mathrm{meV})$} & \multicolumn{2}{|c|}{$K_{e}(\mathrm{D})(\mathrm{meV})$} & \multicolumn{2}{|c|}{$K_{e}(\mathrm{O})(\mathrm{meV})$} \\
\hline & & Expt. & Calc. & Expt. & Calc. & Expt. & Calc. & Expt. & Calc. \\
\hline Ice Ih & 118 & 149 & 144 & 34 & 38.2 & 97 & 100.3 & 43 & 41.4 \\
\hline Vapor & 295 & 135 & 151.8 & $\ldots$ & 52.7 & 100 & 112.7 & $\ldots$ & 57.2 \\
\hline
\end{tabular}
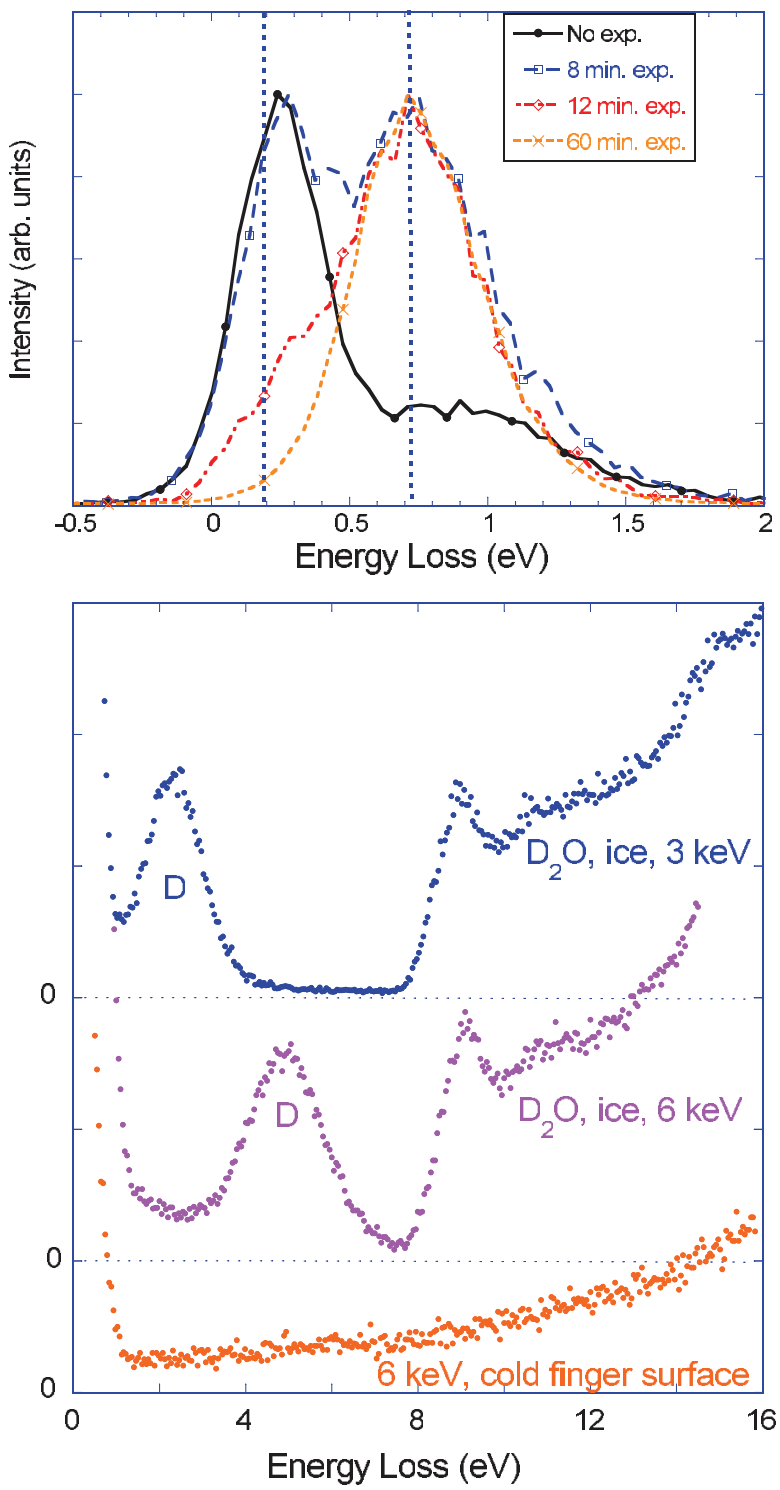

FIG. 2. The top panel shows a sequence of spectra of the low loss region, obtained for electrons scattered from the $\mathrm{Cu}$ cold finger, before and after exposure to $\mathrm{D}_{2} \mathrm{O}$ vapor. The $\mathrm{Cu}$ peak is replaced by an $\mathrm{O}$ peak when the ice grows. The lower panel shows the spectra for up to $16 \mathrm{eV}$ energy loss before water exposure, and after a thick layer of $\mathrm{D}_{2} \mathrm{O}$ ice was grown. Here the zero of the energy scale is aligned with the maximum intensity of the elastic peak. The structure above $8 \mathrm{eV}$ is typical for ice and is completely absent before the water vapor exposure. The peak near 2.5 (3 keV incoming energy) and $5 \mathrm{eV}$ (6 keV incoming energy) is due to electrons scattered from $\mathrm{D}$, and is not seen in low-momentum transfer experiments.

\section{B. Peak separation and peak area ratio}

In Fig. 3 we show the spectra for light and heavy water. For light water we carried out measurements at $1.5 \mathrm{keV}$, $2.5 \mathrm{keV}$, and $3 \mathrm{keV}$, and for heavy water at $3 \mathrm{keV}, 5 \mathrm{keV}$, and $6 \mathrm{keV}$. For the spectra shown side-by-side in Fig. 3, the mean recoil of $\mathrm{H}$ is the same as the mean recoil of $\mathrm{D}$. Spectra for $\mathrm{H}$ and $\mathrm{D}$ at the same recoil values are quite similar, but the $\mathrm{D}$ peak width is noticeable smaller. The smaller width at equal mean recoil energy implies (see Eq. (3)) that the mean kinetic energy of the $\mathrm{D}$ atoms is less than that of the $\mathrm{H}$ atoms.

The H(D)-O separation is, within a few \%, in agreement with the prediction of the very simplistic model that the electron scatters from a free particle (see Tables $\mathrm{V}$ and VI). At first sight this may seem surprising, as the recoil energy is less, or of the order of the $\mathrm{H}-\mathrm{O}$ bond energy $(4.8 \mathrm{eV})$. Within the neutron scattering literature such values of the recoil have been studied extensively, both theoretically and experimentally. ${ }^{3,21,33}$ Indeed it was found that the planewave impulse approximation (PWIA, the approximation that simplifies the collision to that between free particles) can apply even for cases where the recoil energy is not enough to break the chemical bond.

For ice the good agreement for the $\mathrm{O}-\mathrm{H}(\mathrm{D})$ separation indicates also that charging is not a major issue. Charging would change the effective kinetic energy of the scattering electron and hence, result in different values for the $\mathrm{O}-\mathrm{H}(\mathrm{D})$ separation.

According to the theory developed by Sears ${ }^{33}$ the peak shape at lower momentum transfers should not be symmetric, and thus deviate from a simple Gaussian predicted by the PWIA. Sears derived that, in first approximation, the peak shape should resemble the sum of a Gaussian and an impulse correction term that is proportional to the third derivative with respect to energy loss of this Gaussian. At the lower recoil energies, where the corrections to the PWIA are largest, we tried to fit the spectra with and without an impulse correction. The results are shown in Fig. 4 for a $1.5 \mathrm{keV} \mathrm{H}_{2} \mathrm{O}$ and the $3 \mathrm{keV}$ $\mathrm{D}_{2} \mathrm{O}$ measurement. For the $1.5 \mathrm{keV} \mathrm{H}_{2} \mathrm{O}$ measurement and, even more so, the $3 \mathrm{keV} \mathrm{D}_{2} \mathrm{O}$ measurements the fit including impulse correction reproduces the details of the shape of the H(D) peak better than without. As the impulse correction affects also the intensity near the $\mathrm{O}$ peak, the fitting procedure with impulse correction results in a slightly different shape of the $\mathrm{O}$ peak. Generally, the impulse correction results in a somewhat $(3 \%-10 \%)$ smaller area of the $\mathrm{H}$ peak. At higher 


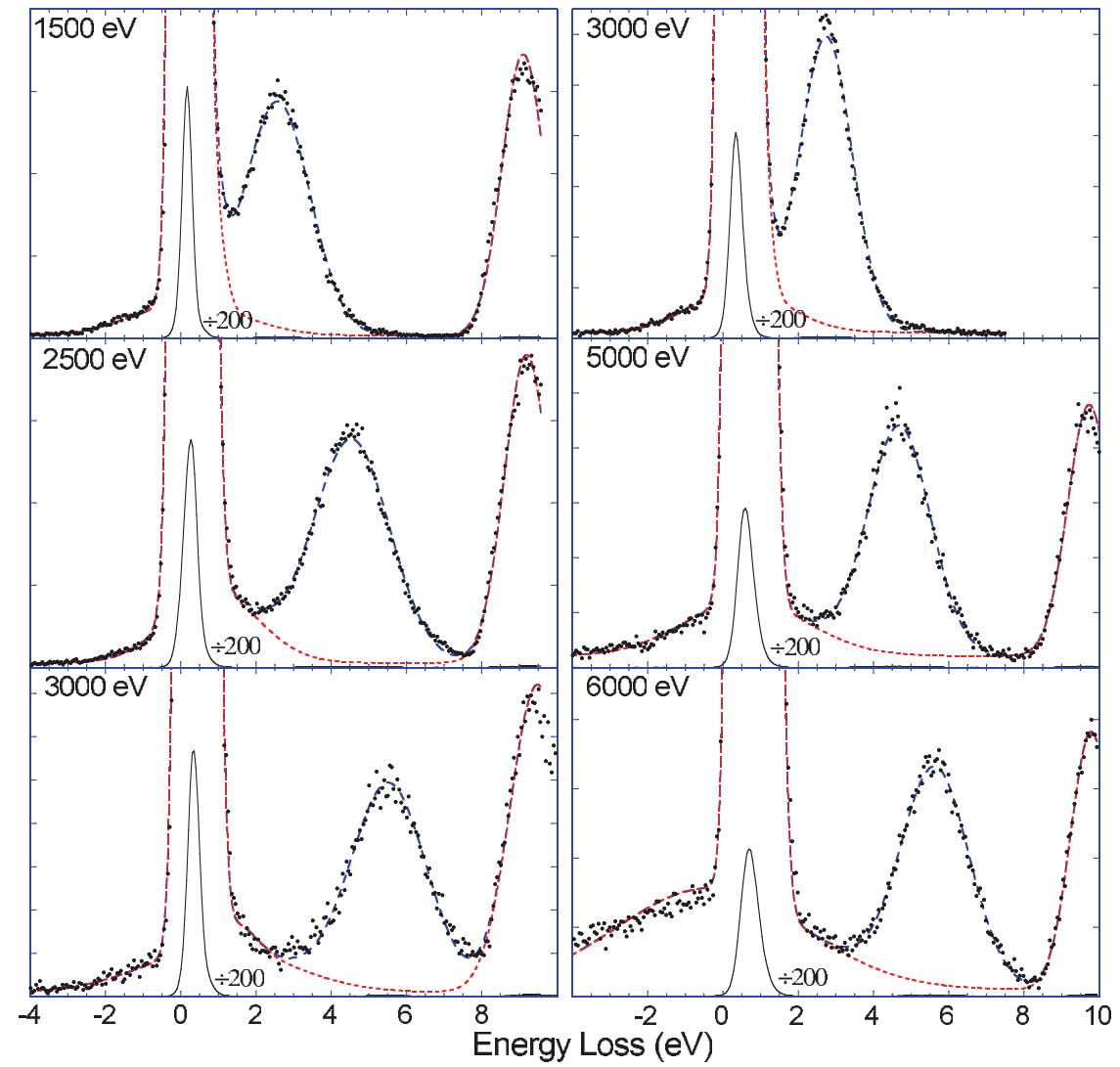

FIG. 3. Spectra obtained from $\mathrm{H}_{2} \mathrm{O}$ and $\mathrm{D}_{2} \mathrm{O}$ ice at the energies as indicated. The peak near zero energy loss is due to electrons scattered from $\mathrm{O}$, the peak moving to higher energy loss with increasing $E_{0}$ is due to electrons scattered from protons or deuterons. The peak near $9 \mathrm{eV}$ is due to electrons scattered elastically from $\mathrm{O}$ and inelastically in the ice layer. The spectra were fitted (blue long dash). The red short dash is the fitted function with the Gaussian describing the $\mathrm{H}$ or $\mathrm{D}$ peak removed.

values of the recoil energies the impulse correction terms were even smaller, and were not considered.

One measurement was done at $6 \mathrm{keV}$ incoming energy for $\mathrm{H}_{2} \mathrm{O}$. This case was even harder to analyse, as the $\mathrm{H}$ peak intensity is now at similar energy losses as the onset of the loss spectrum due to electronic excitations. In Fig. 5 we compare the $6 \mathrm{keV}$ spectrum above $5 \mathrm{eV}$ energy loss with that of the $1.5 \mathrm{keV}$ measurement. In the latter case all intensity above $5 \mathrm{eV}$ is due to electronic excitations. This spectrum was scaled such that the intensity of the $6 \mathrm{keV}$ measurement and the $1.5 \mathrm{keV}$ measurement was the same above $15 \mathrm{eV}$ energy loss.

TABLE V. Calculated O-H separation and $\mathrm{O}: \mathrm{H}$ intensity ratio compared to the calculated ones. The bold numbers are results from ice measurements, the italicized number are obtained from gas phase measurements. The values marked with a $*$, includes final state effects as a fitting parameter.

\begin{tabular}{|c|c|c|c|c|}
\hline $\begin{array}{l}E_{0} \\
(\mathrm{keV})\end{array}$ & $\begin{array}{l}\text { H-O sep. } \\
\text { obs. }(e V)\end{array}$ & $\begin{array}{l}\text { H-O sep. } \\
\text { calc. }(e V)\end{array}$ & $\begin{array}{l}\text { Ratio } \\
\text { obs. }\end{array}$ & $\begin{array}{l}\text { Ratio } \\
\text { calc. }\end{array}$ \\
\hline 1.5 & $2.46,2.56^{*}$ & 2.62 & $37.2,39.4 *$ & 37.5 \\
\hline 2 & $3.48,3.53^{*}$ & 3.49 & $35.2,35.4^{*}$ & 36.4 \\
\hline 2.5 & 4.36 & 4.37 & 38.2 & 35.5 \\
\hline 3 & $5.34, \mathbf{5 . 2 7}$ & 5.24 & $39.8, \mathbf{3 6 . 6}$ & 34.9 \\
\hline 6 & 10.89 & 10.51 & 40.8 & 33.3 \\
\hline
\end{tabular}

If one assumes that the shape of the energy loss spectrum due to electronic excitations does not depend on the incoming energy, one can obtain in this way the shape of the $6 \mathrm{keV} \mathrm{H}$ peak by a subtraction. Indeed the difference of these two spectra (see Fig. 5) is reasonably close to a Gaussian distribution, but the error bars at higher energy losses are quite large. A fit gives a center of the distribution at $0.4 \mathrm{eV}$ larger energy loss than what is calculated, assuming the collision between free particles, but, considering the complexity of the background subtraction and limited statistics, this is as good an agreement as can be reasonably expected.

In spite of the fact that the $\mathrm{H}$ peak appears quite well resolved, determining the precise ratio of the area of the $\mathrm{H}$ and $\mathrm{O}$ peaks is surprisingly hard. This applies in particular to the gas phase measurements where the statistics were not as good

TABLE VI. Same as Table V, but for $\mathrm{D}_{2} \mathrm{O}$.

\begin{tabular}{lcccc}
\hline \hline $\begin{array}{l}E_{0} \\
(\mathrm{keV})\end{array}$ & $\begin{array}{l}\text { D-O sep. } \\
\text { obs. (eV) }\end{array}$ & $\begin{array}{l}\text { D-O sep. } \\
\text { calc. }(\mathrm{eV})\end{array}$ & $\begin{array}{l}\text { Ratio } \\
\text { obs. }\end{array}$ & $\begin{array}{c}\text { Ratio } \\
\text { calc. }\end{array}$ \\
\hline 3 & $2.45,2.58^{*}$ & 2.45 & $\begin{array}{l}32.6,36.2^{*} \\
\text { (2.6.38.1* }\end{array}$ & 34.9 \\
5 & $\mathbf{2 . 4 4}, \mathbf{2 . 5 2}$ & & $\mathbf{3 7 . 2}$ & 33.6 \\
6 & $\mathbf{4 . 0 5}$ & 4.08 & $\mathbf{3 7 . 3}$ & 33.3 \\
\hline \hline
\end{tabular}




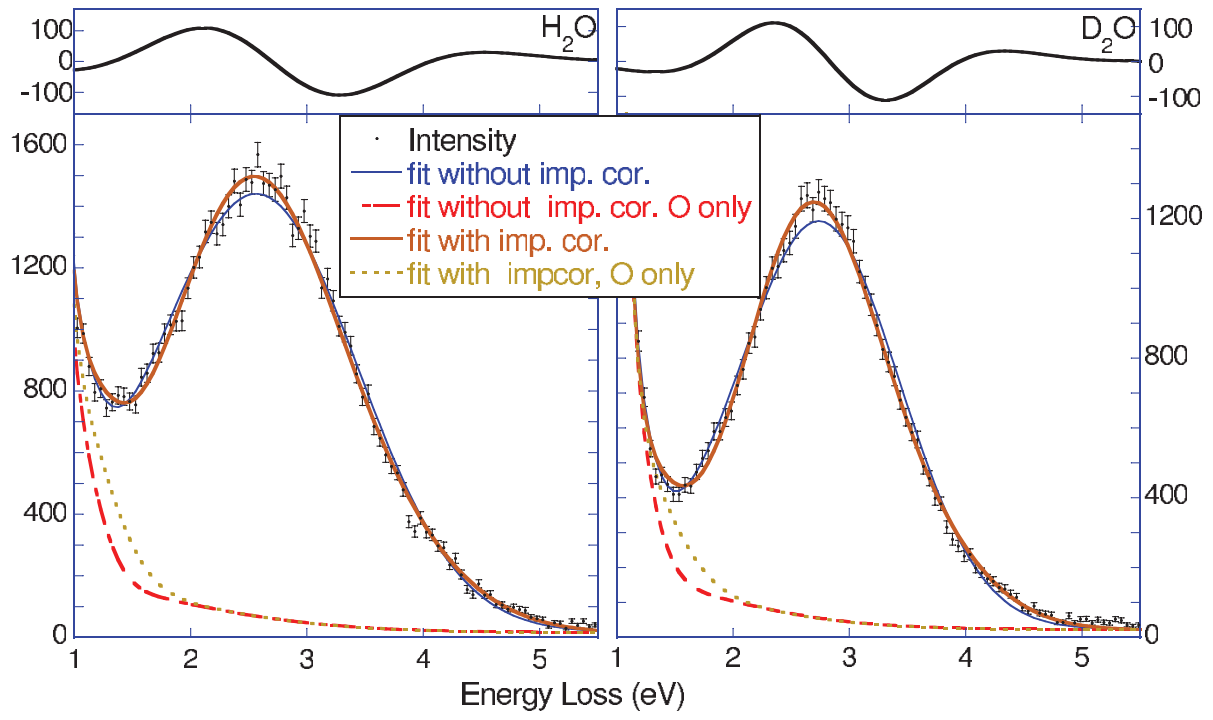

FIG. 4. Detailed analysis of the $\mathrm{H}$ peak shape for $E_{0}=1.5 \mathrm{keV}$ and the $\mathrm{D}$ for $E_{0}=3.0 \mathrm{keV}$. The peaks were fitted with a single Gaussian (marked "without imp cor") and a single Gaussian plus its third derivative (marked "imp cor"). The magnitude of the correction term is plotted in the top panel using the same vertical scale. The fit including the impulse correction term describes the peak shape clearly better, especially for $\mathrm{D}_{2} \mathrm{O}$. The corresponding shapes obtained for the oxygen peak are shown as well. The areas, separation, and peak width obtained with impulse correction are reproduced in Tables V, VI, and VII marked with a*.

as for ice. The $\mathrm{O}: \mathrm{H}$ area ratio varies by about $10 \%$, somewhat more for the gas-phase measurements, depending on the assumptions one makes in fitting the $\mathrm{O}$ peak. Agreement with theory is reasonable (see Tables $\mathrm{V}$ and VI) but on average the experimentally obtained $\mathrm{O}: \mathrm{H}$ area ratio is higher by $\approx 10 \%$ than the calculated one and is equal to that of the measured $\mathrm{O}: \mathrm{D}$ area ratio. It should be stressed that the differential cross section calculations have been tested experimentally mainly at lower momentum transfer ( 15 a.u. and below ${ }^{34}$ ) compared to values of 19-40 a.u used in the present experiments. Thus overall the agreement is quite satisfactory. The largest deviation $(\approx 20 \%)$ was found for the $6 \mathrm{keV} \mathrm{H}_{2} \mathrm{O}$ case. This is not surprising, in the light of of the more problematic background subtraction procedure required here. In no case did the difference between theory and experiment reach $30 \%$, the value often seen for anomalous intensities for neutron scattering from H. ${ }^{8,14,19}$

\section{C. $H(D)$ peak width}

From the measured width, as obtained from the fit, we subtract in quadrature our estimate of the energy resolution $\left(\sigma_{\text {res }} \approx 0.2 \mathrm{eV}\right)$. This is only a small correction for the $\mathrm{H}$ and $\mathrm{D}$ width. The resulting widths are shown in Table VII and compared to the calculated width based on Eq. (3), and kinetic energies of $\mathrm{H}$ and $\mathrm{D}$ as given in Table IV.

For the ice measurements, where the statistics were quite good, the agreement between measured and calculated width was better than $5 \%$ and the experimentally obtained width scatter around the calculated ones. The largest deviation was found for the $3 \mathrm{keV} \mathrm{H}_{2} \mathrm{O}$ vapor measurement where the observed width was $10 \%$ less than the calculated one. If we take $5 \%$ as the accuracy of the width measurement, then, due to the nature of Eq. (3), this method should be able to determine the kinetic energy of H,D with an accuracy of only $10 \%$.

TABLE VII. Calculated recoil energy and intrinsic width using the kinetic energy for $\mathrm{H}$ and $\mathrm{D}$ as given in Table IV. The bold numbers are for ice (at $118{ }^{\circ} \mathrm{K}$ ), the italicized number for gas phase measurements (at room temperature). For the $1.5,2.0 \mathrm{keV} \mathrm{H}_{2} \mathrm{O}$ and $3 \mathrm{keV} \mathrm{D}_{2} \mathrm{O}$ case the value marked with a $*$, includes final state effects as a fitting parameter.

\begin{tabular}{|c|c|c|c|c|c|c|}
\hline $\begin{array}{l}E_{0} \\
(\mathrm{keV})\end{array}$ & $\begin{array}{l}\text { Recoil } \\
\mathrm{H}(\mathrm{eV})\end{array}$ & $\begin{array}{c}\sigma \mathrm{H} \\
\text { calc. }(\mathrm{eV})\end{array}$ & $\begin{array}{c}\sigma \mathrm{H} \\
\text { obs. (eV) }\end{array}$ & $\begin{array}{c}\text { Recoil D } \\
(\mathrm{eV})\end{array}$ & $\begin{array}{c}\sigma \mathrm{D} \\
\text { calc. }(\mathrm{eV})\end{array}$ & $\begin{array}{c}\sigma \mathrm{D} \\
\text { obs. }(\mathrm{eV})\end{array}$ \\
\hline 1.5 & 2.79 & $\mathbf{0 . 7 3}, 0.75$ & $\mathbf{0 . 8 3}, 0.76 *, 0.74$ & 1.40 & & \\
\hline 2.0 & 3.72 & $\mathbf{0 . 8 5}, 0.87$ & $0.830 .82 *$ & 1.86 & & \\
\hline 2.5 & 4.66 & $\mathbf{0 . 9 5}, 0.97$ & 0.99 & 2.33 & & \\
\hline 3 & 5.59 & $\mathbf{1 . 0 3}, 1.05$ & $\mathbf{1 . 0 4}, 0.93$ & 2.80 & $\mathbf{0 . 6 1}, 0.65$ & $0.66,0.61^{*}$ \\
\hline 3 & & & & & & $0.66,0.60^{*}$ \\
\hline 5 & 9.34 & & & 4.67 & $\mathbf{0 . 7 9}, 0.84$ & 0.80 \\
\hline 6 & 11.22 & $\mathbf{1 . 4 7 ,} 1.50$ & 1.44 & 5.61 & $\mathbf{0 . 8 7}, 0.92$ & 0.88 \\
\hline
\end{tabular}



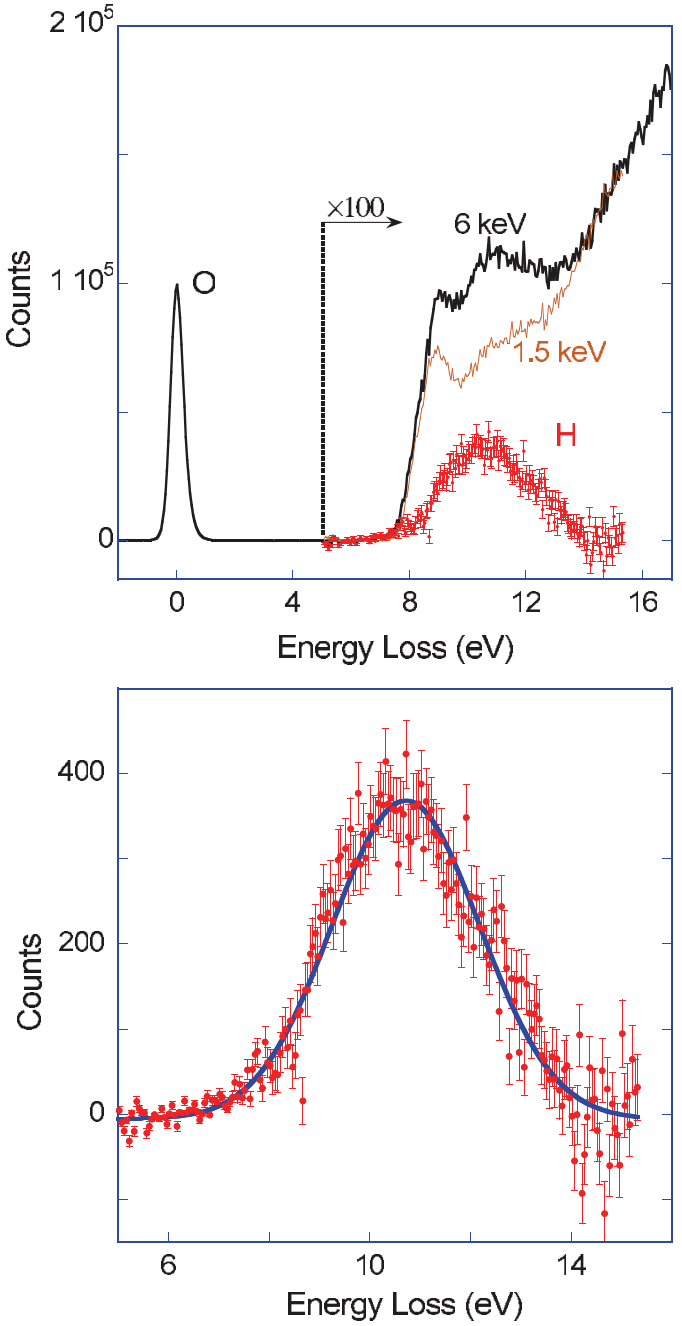

FIG. 5. Analysis of the $6 \mathrm{keV} \mathrm{H}_{2} \mathrm{O}$ measurement. Here the energy loss structure and the proton elastic peak overlap. The energy loss structure, as measured at $1.5 \mathrm{keV}$ was scaled so to coincide with the $6 \mathrm{keV}$ spectrum at 14-16 eV energy loss. The contribution due to electrons scattered from hydrogen is obtained by subtracting the $6 \mathrm{keV}$ and $1.5 \mathrm{keV}$ spectra as indicated. The resulting spectrum resembles a Gaussian, with a width, position, and area as summarised in Tables V and VII.

We extracted our best estimate of the H (D) kinetic energy, based on all measurements in Table VII, and reproduced that in Table IV. This average value of the mean kinetic energy (based on several measurements at different energies) agrees with the theoretical one on a $5 \%$ level for the ice measurements. For the gas-phase measurement the agreement is somewhat less good (mean kinetic energy appears $15 \%$ too low). We attribute that, at least in part, due to the poorer statistics, and the more involved background subtraction procedure required here, as well as the more limited dataset available.

\section{O peak width}

We measured the $\mathrm{O}$ width for a high incoming energy value of $6 \mathrm{keV}$ to maximise the effect of Doppler broadening. Due to its small intrinsic width the resolution of our spectrometer is a critical factor. To obtain the best resolution we measured the spectrum with the incoming energy fixed, i.e.,

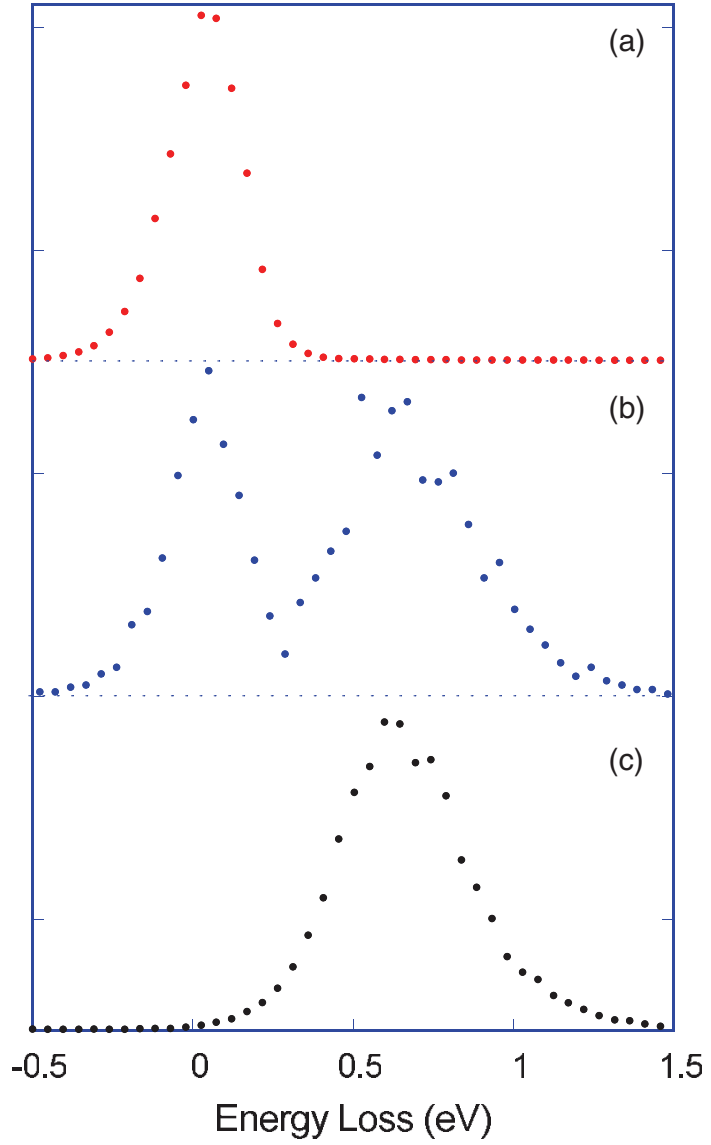

FIG. 6. (a) The spectrum of $6 \mathrm{keV}$ electrons scattered from the Au coated cold finger. We take its peak shape (due to electrons backscattered from $\mathrm{Au}$ ) as a signature of the spectrometer resolution. In (b) we show a spectrum taking over a short time during the initial stages of the $\mathrm{H}_{2} \mathrm{O}$ overlayer growth on the Au film. In (c) we show the spectrum for a thick ice layer. The Au signal is now completely attenuated by the ice overlayer.

we did not scan the area of interest over the two-dimensional detector. This removes the slight deterioration of the resolution due to imperfections in the calibration of the energy scale of the detector.

Now it is imperative to have the best estimate possible of the systems energy resolution, as the $\mathrm{O}$ width exceeds the energy resolution by only a factor of $\approx 2$, even at high $E_{0}$ values. The observed actual $\mathrm{Cu}$ width before ice growth (see Fig. 2) is $0.35 \mathrm{eV} \mathrm{FWHM}(\sigma=0.15 \mathrm{eV})$ can be used as an estimate of the experimental resolution. However even the $\mathrm{Cu}$ elastic peak is Doppler broadened. The kinetic energy of $\mathrm{Cu}$ at room temperature is larger than expected from purely classical arguments $\left(\frac{3}{2} k T\right)$ as quantum effects contribute even at room temperature. ${ }^{35}$ A good estimate can be obtained from lattice dynamics simulation packages, and we obtain $41 \mathrm{meV}$ using the GULP $\operatorname{code}^{36}$ at room temperature, reducing to $21 \mathrm{meV}$ at $120 \mathrm{~K}$. From Eq. (3) we obtain a value of $100 \mathrm{meV}(\sigma)$ for the intrinsic width of $\mathrm{Cu}$ at $6 \mathrm{keV}$ incoming energy at RT. Using this line of argument we estimate the energy resolution of the spectrometer (without "scanning") to be $\sigma_{\text {res }}=0.10 \mathrm{eV}$, an extremely low value for an experiment using a $\mathrm{BaO}$ cathode as an emitter. 
The best estimate is obtained using a pure, heavy target, as Doppler broadening is smallest in that case. To accomplish this we evaporated $\mathrm{Au}$ on the cold finger and inserted it quickly in the gas-phase spectrometer. The elastic peak shows now only one component (see Fig. 6), whereas the cold finger itself showed, besides $\mathrm{Cu}$, clearly the presence of $\mathrm{O}$ (and possibly $C$ ) atoms at the surface (see Figure 2$)$. The width $(\sigma)$ of the Au peak was $0.11 \mathrm{eV}$, indeed somewhat narrower than the $\mathrm{Cu}$ peak and in fair agreement with the resolution estimate of the spectrometer, based on the calculated $\mathrm{Cu}$ kinetic energy. Note that this is a very favorable case: only very limited beam current is required (less than $1 \mathrm{nA}$ ) and at relatively high energies. This makes it possible to reduce the filament temperature somewhat, and space charge effects should be very small for these energetic low-intensity beams

The energy scale of the spectrum was converted to a momentum scale (referred to as $y$-scaling in the neutron literature ${ }^{33}$ ), by subtracting $q^{2} / 2 M$ from the energy and subsequently multiplying the energy loss scale by $M / q$. The line shape was then calculated using two models:

(a) fitting the spectrum with a Gaussian plus correction terms proportional to the 3rd and 4th derivative of this Gaussian. This is based on the series expansion developed by Sears (b) fitting the spectrum with a Gaussian plus correction terms proportional to the 3 rd derivative of this Gaussian plus the first non-zero correction term of the Hermite polynomial expansion series (i.e., the $\mathrm{H}_{4}$ term), that takes into account a nonGaussian target momentum distribution (see, for example, Eq. (4.19) of Ref. 37). Next the line shape was convoluted with the detector response function. The detector response function was derived from a two-component Gaussian fit of the Au spectrum, and a nonlinear least square fitting routine was used to obtain the best description of the measurement. The best fit obtained with both models is shown in Fig. 7 as well as the magnitude of the correction terms used.

Procedure (a) gives a somewhat better description of the experiment than procedure (b) and procedure (a) was used to extract the kinetic energy of $\mathrm{O}$. Whether the magnitude of the correction term was in-line with the theory of Sears was not investigated. Our main focus was getting a good description of the second moment of the distribution. The second moment of either procedure was larger than that obtained when fitting the spectrum with a plain Gaussian. From the second moment the mean kinetic energy is straightforwardly evaluated. The results are reproduced in Table IV for both $\mathrm{H}_{2} \mathrm{O}$ and $\mathrm{D}_{2} \mathrm{O}$. Considering that the intrinsic $\mathrm{O}$ width is of the order of the energy resolution, the agreement between experiment and theory is as good as can be expected.

\section{DISCUSSION AND CONCLUSION}

This is our first attempt to study electron scattering at high momentum transfer from $\mathrm{H}_{2} \mathrm{O}$. There appears little doubt that, just as in the case of $\mathrm{H}_{2}, \mathrm{D}_{2},{ }^{38} \mathrm{CH}_{4},{ }^{2}$ graphite, and diamond, ${ }^{9}$ the measured spectra can be interpreted as a Compton profile of atomic motion, in a way very similar to the interpretation of neutron Compton scattering measurements. The energy separation of the H(D)-O recoil peak is obtained with an accuracy of $2 \%$. The widths of the $\mathrm{H}$ and $\mathrm{D}$ peaks are

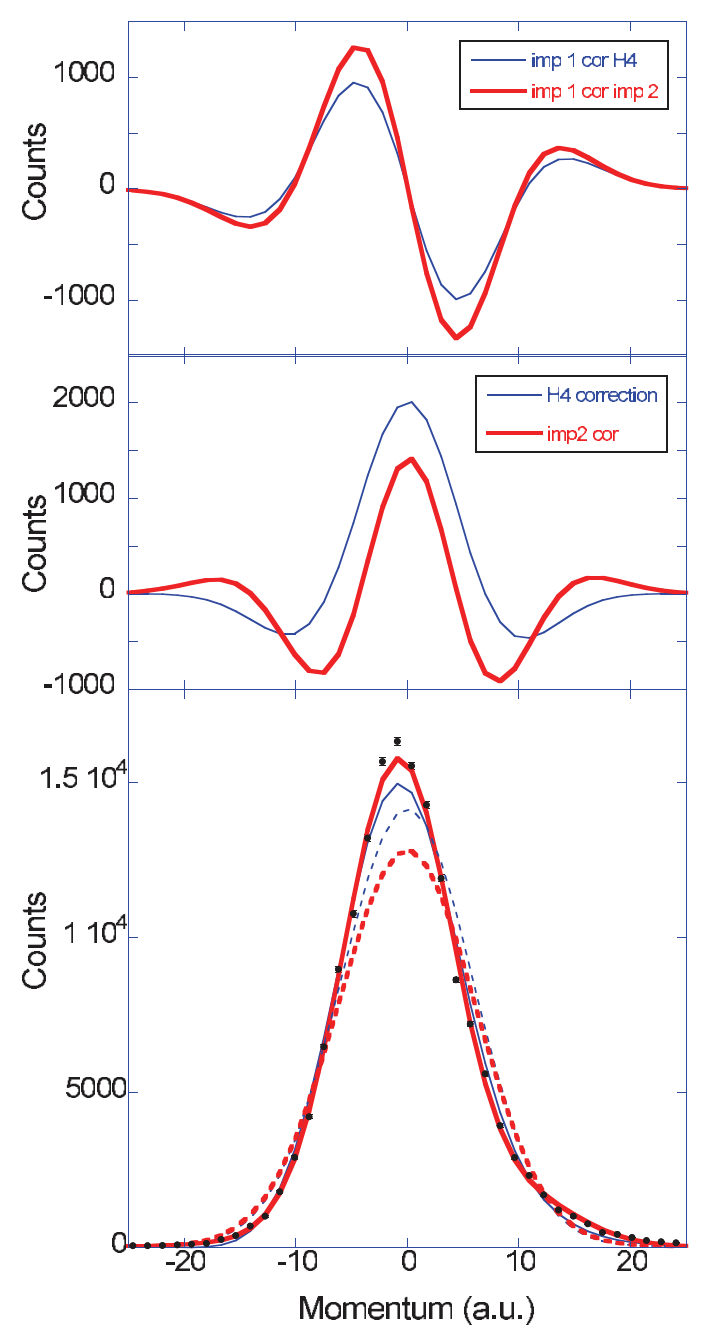

FIG. 7. The lower panel shows an $\mathrm{O}$ elastic peak $\left(E_{0}=6 \mathrm{keV}, \mathrm{H}_{2} \mathrm{O}\right.$, ice $)$ after converting the energy loss scale into a momentum scale, the upper panels show the magnitude of the correction terms. The spectrum (dots) is fitted (thick line) by a sum of a Gaussian (thick, dashed) and two impulse correction terms (thick lines in top and central panel), or by a Gaussian, one impulse correction term and an expansion based on a non-Gaussian $\mathrm{O}$ momentum distribution (thin (blue) lines). The fit based on two impulse correction terms is somewhat better than the one based one impulse correction term plus nonGaussian momentum distribution.

within 5\% in agreement with theoretical estimates. When the average recoil energy is low, then inclusion of final state effects improves the quality of the fit, with only minor changes in the extracted values of the mean kinetic energy.

A more careful interpretation is required for the width of the $\mathrm{O}$ peak. As predicted by the theory, the $\mathrm{O}$ width in $\mathrm{D}_{2} \mathrm{O}$ is found experimentally to be larger than the $\mathrm{O}$ width in $\mathrm{H}_{2} \mathrm{O}$. However, when fitting the spectrum with a simple Gaussian line shape, the obtained $\mathrm{O}$ kinetic energies are smaller than expected for both $\mathrm{H}_{2} \mathrm{O}$ and $\mathrm{D}_{2} \mathrm{O}$. When fitting with more elaborate peak shapes the agreement between the experimental and calculated kinetic energy improves. Good agreement is obtained when fitting with first and second order impulse correction terms. Alternatively one can assume a non-Gaussian momentum distribution and obtain a fit using a Hermite expansion as described in, e.g., Ref. 37. Both correction procedures produce reasonable fits and values for the mean kinetic 

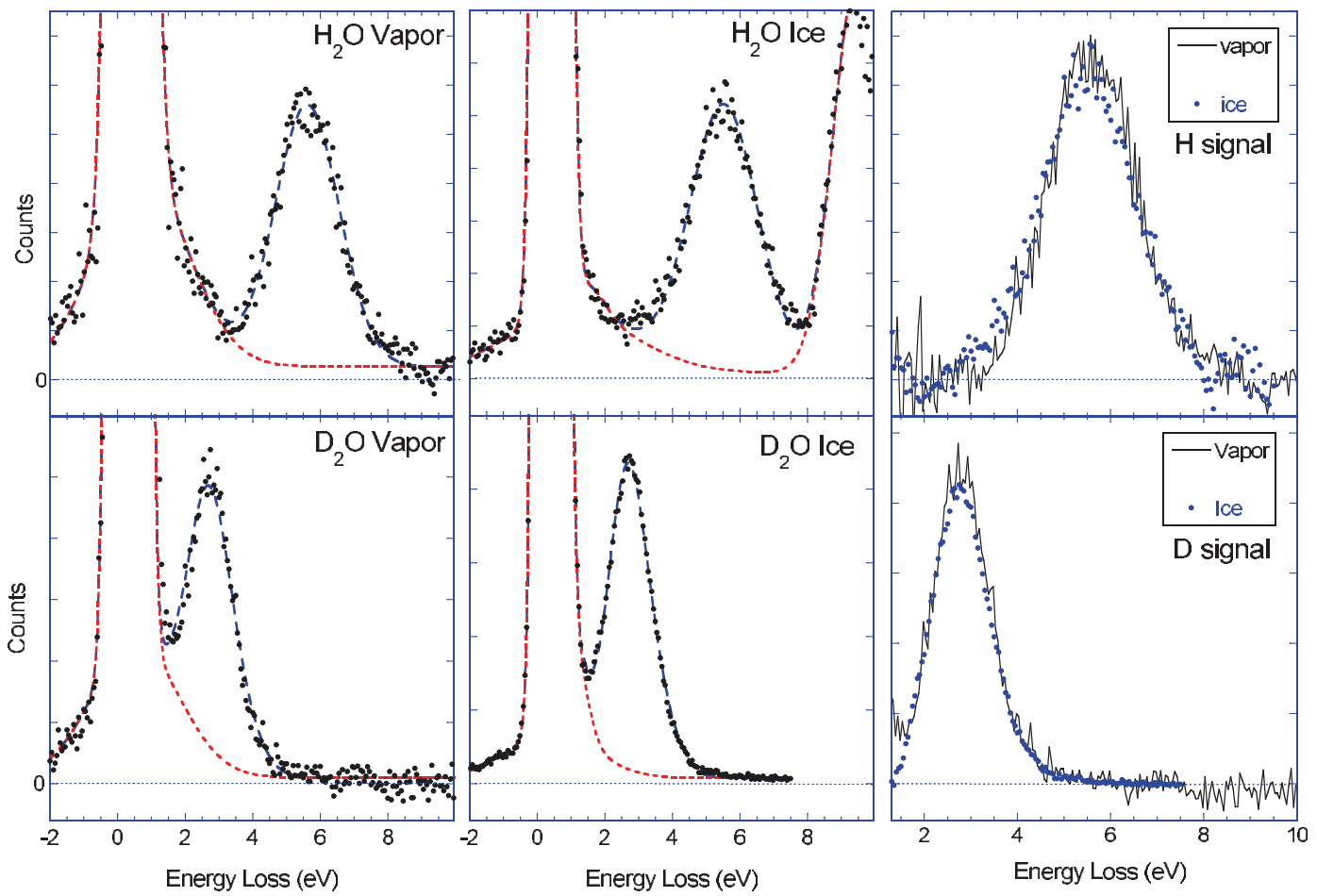

FIG. 8. A comparison of the $3 \mathrm{keV}$ measurement of ice and water vapor for $\mathrm{H}_{2} \mathrm{O}$ (top) and $\mathrm{D}_{2} \mathrm{O}$ (bottom). The $\mathrm{O}$ peak area is normalised to the same value for all measurements. The fit of the spectra (blue, dashed line) and the value of this fit with the Gaussian, describing the H (or D) peak, removed (red, short-dashed line) is shown as well. The right panel shows the intensity H (D) above this red dashed line for both vapor and ice. Both curves are remarkably similar in shape and area.

energy. In principle we can distinguish between both options by increasing the incoming energy $E_{0}$ significantly, but our spectrometer is not designed to do that. If the correction term decreases with increasing $E_{0}$, then the impulse correction interpretation is correct. If the correction term persists with increasing $E_{0}$, then we have to attribute it to a non-Gaussian momentum distribution.

The peak area ratio is somewhat harder to determine than the peak width. Here we have an error of $10 \%$. In some problematic cases, e.g., gas phase measurements with poorer statistics, and the $6 \mathrm{keV} \mathrm{H}_{2} \mathrm{O}$ ice measurement requiring background subtraction, the experimental ratio can differ by more than $20 \%$ from the calculated one. All large deviations are for cases where the relative $\mathrm{H}$ area is smaller than expected. One interpretation would be that the calculated cross section ratio is not quite right. It could also be seen as a sign of "anomalous" scattering behavior, related to decoherence, as has been discussed extensively in the NCS literature and used to explain the puzzling H/D peak area ratios in mixtures of heavy and light water ${ }^{14}$ or the electron scattering result from $\mathrm{H}_{2}-\mathrm{D}_{2}$ mixtures. ${ }^{39,40}$ In this "anomalous" scattering framework the oxygen to hydrogen area ratio are expected to differ for $\mathrm{H}_{2} \mathrm{O}$ and $\mathrm{D}_{2} \mathrm{O}$. The average deviation of the observed $\mathrm{O} / \mathrm{H}$ peak area ratio from the calculated ratio for the measurements of Table $\mathrm{V}$ is a factor 1.1 (using the analysis including final state effects). In the same way the O/D peak area ratio deviates from the calculated ratio is also a factor 1.1 for the measurements in Table VI. Thus for water we do not find any evidence that the cross section of $\mathrm{H}$ and $\mathrm{D}$ differ. For a discussion of electron scattering experiments of $\mathrm{H}_{2}, \mathrm{D}_{2}$ see Refs. 38 and 41.
Let us further compare for this reason the spectra of vapor and ice, taken at $3 \mathrm{keV}$, for both $\mathrm{H}_{2} \mathrm{O}$ and $\mathrm{D}_{2} \mathrm{O}$. Here we would expect the interaction with the environment (and hence decoherence) to be much stronger in the case of ice than in the case of isolated molecules. The spectra, normalised to equal $\mathrm{O}$ peak area are shown in Fig. 8. The background below the $\mathrm{H}$ peak is determined including, for the case of ice, also a fit for the onset of the inelastic excitations. The $\mathrm{H}$ peak above the background is plotted in the right panel where we compare ice and vapor directly. The $\mathrm{H}$ peak appears somewhat narrower for the vapor than for the ice measurement, explaining the $10 \%$ difference in peak area ratio obtained. However, overall the measurements are very similar in both vapor and ice, for both $\mathrm{H}_{2} \mathrm{O}$ and $\mathrm{D}_{2} \mathrm{O}$. From a decoherence-point-of-view ice and vapor should be completely different.

Thus the present measurements on $\mathrm{H}_{2} \mathrm{O}$ and $\mathrm{D}_{2} \mathrm{O}$ do not show any indication for anomalous scattering from protons, nor does it point to any influence of decoherence on the outcome of the measurement. Hence, in contrast to neutron scattering from liquid samples of $\mathrm{H}_{2} \mathrm{O}$ and $\mathrm{D}_{2} \mathrm{O},{ }^{14}$ there are no anomalies in the present experiment.

According to Ref. 42 the occurrence of anomalous neutron scattering from $\mathrm{H}$ in $\mathrm{H}_{2} \mathrm{O}$ requires that the coherence length to be larger than the distance between the two protons $d_{\mathrm{HH}}=1.6 \AA$, in $\mathrm{H}_{2} \mathrm{O}$. The coherence length is defined as: $l_{c}$ $=\lambda_{n} E_{r} / \Delta E_{r}$ where $\lambda_{n}$ is the de-Broglie wavelength of the incident neutrons, $E_{r}$ is the resonance energy, and $\Delta E_{r}$ is the width of the resonance level of gold filter used in the neutron measurement. ${ }^{14}$ This yields $l_{c}=2.35 \AA$ which is larger than $d_{\mathrm{HH}}$ thus fulfilling the condition of Ref. 42 for the occurrence 
of the anomaly. For electron scattering, a similar coherence length was defined, ${ }^{19}$ namely $l_{e}=\lambda_{e} E_{0} / \Delta E_{e}$, where $\lambda_{e}$ the de-Broglie wavelength of electrons with an incident energy $E_{0}$. Here $\Delta E_{e}$ is determined by the energy spread of the gun emitting the electrons in our case $\Delta E_{e} \approx 0.3 \mathrm{eV}$. Thus for $E_{0}=3 \mathrm{keV}$ one gets $\lambda_{e}=0.22 \AA$ and $l_{e}=2200 \AA \gg d_{\mathrm{HH}}$. This means that the coherence length condition is fulfilled for $3 \mathrm{keV}$ electrons striking an $\mathrm{H}_{2} \mathrm{O}$ sample, but no anomalous scattering from $\mathrm{H}$ was observed. It may also be added that a recent neutron scattering study from $\mathrm{H}$ in a water sample using a direct geometry spectrometer did not reveal any anomalous drop in the scattering intensity ${ }^{43}$ and agrees with the standard cross section. This result was criticized in Ref. 44 on the grounds that the energy resolution of the direct geometry spectrometer ${ }^{43}$ is far inferior to that of the inverse geometry spectrometer used in Ref. 44. It was also argued ${ }^{43}$ that the deficit in the scattering intensity is inconsistent with the sum rule of neutron scattering as also mentioned in Ref. 15: if there is a deficiency in intensity in an interference process, the missing part should reappear somewhere to conserve the sum rule. The missing intensity was never found in Ref. 44 or in any previous work supporting the existence of the anomaly.

In conclusion electron scattering at high momentum transfer provides direct information about the mass of the elements present. The width of the peak reflects the kinetic energy of the scattering atom. For the $\mathrm{H}$ and in particular the $\mathrm{D}$ peak in water we see deviations at low incoming energy from a Gaussian line shape, in line with the presence of final state effects. In this first attempt we were successful in observing the difference in kinetic energy of $\mathrm{O}$ in light and heavy water. Also it is clear that the kinetic energy of $\mathrm{D}$ in $\mathrm{D}_{2} \mathrm{O}$ is less than that of $\mathrm{H}$ in $\mathrm{H}_{2} \mathrm{O}$. The calculated energy separations of the recoil peaks is reproduced by the measurement with a precision of $2 \%$. The width of the $\mathrm{H}$ and $\mathrm{D}$ peaks agrees in most cases with the calculated ones with an accuracy of $5 \%$. The extraction of the $\mathrm{O}$ mean kinetic energy from the data requires great care. The assumption of a Gaussian peak shape fails here. The second moment extracted from a careful fit of the data, going beyond a simple Gaussian fit is in good agreement with the expected one, based on the mean kinetic energy of $\mathrm{O}$ in ice.

The O:H and O:D area ratio are identical and on average slightly $(10 \%)$ larger than predicted by theory. Vapor and ice spectra are very similar. This similarity indicates that multiple elastic scattering, expected to occur in solids for a significant fraction of the trajectories, does not affect the shape of the spectra much, a conclusion also reached in our previous study of Au overlayers on graphite. ${ }^{9}$

\section{ACKNOWLEDGMENTS}

This work was made possible by financial support of the Australian Research Council. One of us (R.M.) would like to thank Professor F. Yubero and Professor K. Tokesi for helpful correspondence.
${ }^{1}$ M. Vos, M. Went, G. Cooper, and C. Chatzidimitriou-Dreismann, J. Phys. B 41, 135204 (2008).

${ }^{2}$ M. Vos, J. Chem. Phys. 132, 074306 (2010).

${ }^{3}$ C. Andreani, D. Colognesi, J. Mayers, G. F. Reiter, and R. Senesi, Adv. Phys. 54, 377 (2005).

${ }^{4}$ O. Shahal and R. Moreh, Phys. Rev. Lett. 40, 1714 (1978).

${ }^{5}$ R. Moreh, W. C. Sellyey, D. Sutton, and R. Vodhanel, Phys. Rev. C 31, 2314 (1985).

${ }^{6}$ R. Moreh, O. Beck, D. Jäger, Y. Finkelstein, U. Kneissl, J. Margraf, H. Maser, and H. H. Pitz, Phys. Rev. B 56, 187 (1997).

${ }^{7}$ M. Vos, Phys. Rev. A 65, 012703 (2001).

${ }^{8}$ C. A. Chatzidimitriou-Dreismann, M. Vos, C. Kleiner, and T. Abdul-Redah, Phys. Rev. Lett. 91, 057403 (2003).

${ }^{9}$ M. Vos, R. Moreh, and K. Tőkési, J. Chem. Phys. 135, 024504 (2011).

${ }^{10}$ C. Pantalei, A. Pietropaolo, R. Senesi, S. Imberti, C. Andreani, J. Mayers, C. Burnham, and G. Reiter, Phys. Rev. Lett. 100, 177801 (2008).

${ }^{11}$ A. Pietropaolo, R. Senesi, C. Andreani, A. Botti, M. A. Ricci, and F. Bruni, Phys. Rev. Lett. 100, 127802 (2008).

${ }^{12}$ D. Flammini, A. Pietropaolo, R. Senesi, C. Andreani, F. McBride, A. Hodgson, M. A. Adams, L. Lin, and R. Car, J. Chem. Phys. 136, 024504 (2012).

${ }^{13}$ F. Yubero and K. Tökèsi, Appl. Phys. Lett. 95, 084101 (2009).

${ }^{14}$ C. A. Chatzidimitriou-Dreismann, T. Abdul-Redah, R. M. F. Streffer, and J. Mayers, Phys. Rev. Lett. 79, 2839 (1997).

${ }^{15}$ J. J. Blostein, J. Dawidowski, S. A. Ibez, and J. R. Granada, Phys. Rev. Lett. 90, 105302 (2003).

${ }^{16}$ J. J. Blostein, L. A. R. Palomino, and J. Dawidowski, Phys. Rev. Lett. 102, 097401 (2009).

${ }^{17}$ R. Moreh, R. C. Block, Y. Danon, and M. Neumann, Phys. Rev. Lett. 94, 185301 (2005).

${ }^{18}$ R. Moreh, R. C. Block, Y. Danon, and M. Neuman, Phys. Rev. Lett. 96, 055302 (2006).

${ }^{19}$ M. Vos, C. A. Chatzidimitriou-Dreismann, T. Abdul-Redah, and J. Mayers, Nucl. Instrum. Methods Phys. Res. B 227, 233 (2005).

${ }^{20}$ L. van Hove, Phys. Rev. 95, 249 (1954).

${ }^{21}$ G. I. Watson, J. Phys.: Condens. Matter 8, 5955 (1996).

${ }^{22}$ F. Salvat, A. Jablonski, and C. J. Powell, Comput. Phys. Commun. 165, 157 (2005).

${ }^{23}$ C. Moore, Atomic Energy Levels (U.S. Department of Commerce, 1971).

${ }^{24}$ R. Moreh and D. Nemirovsky, J. Chem. Phys. 133, 084506 (2010).

${ }^{25}$ G. Herzberg, Infrared and Raman Spectra (Van Nostrand Reinold, New York, 1945).

${ }^{26}$ J. E. Bertie and E. Whalley, J. Chem. Phys. 40, 1637 (1964).

${ }^{27}$ M. J. Taylor and E. Whalley, J. Chem. Phys. 40, 1660 (1964).

${ }^{28}$ E. Zheligovskaya, J. Struct. Chem. 49, 459 (2008).

${ }^{29}$ R. E. Shawyer and P. Dean, J. Phys. C 5, 1028 (1972).

${ }^{30}$ G. S. Fanourgakis and S. S. Xantheas, J. Chem. Phys. 124, 174504 (2006).

${ }^{31}$ M. Vos and M. R. Went, Surf. Sci. 601, 1536 (2007).

${ }^{32}$ R. D. Leapman and S. Sun, Ultramicroscopy 59, 71 (1995).

${ }^{33}$ V. F. Sears, Phys. Rev. B 30, 44 (1984).

${ }^{34}$ F. Salvat, Phys. Rev. A 68, 012708 (2003).

${ }^{35}$ R. Moreh, O. Shahal, and I. Jacob, Nucl. Phys. A 228, 77 (1974).

36. Gale and A. Rohl, Mol. Simul. 29, 291 (2003).

${ }^{37}$ J. Mayers and G. Reiter, Meas. Sci. Technol 23, 045902 (2012).

${ }^{38}$ M. Vos and M. Went, J. Phys. B 42, 065204 (2009).

${ }^{39}$ G. Cooper, A. P. Hitchcock, and C. A. Chatzidimitriou-Dreismann, Phys. Rev. Lett. 100, 043204 (2008).

${ }^{40}$ A. Hitchcock, G. Cooper, R. Bonham, and C. Chatzidimitriou-Dreismann, J. Electron Spectrosc. Relat. Phenom. 181, 135 (2010).

${ }^{41}$ R. Moreh, Nucl. Instrum. Methods Phys. Res. B 279, 49 (2012).

${ }^{42}$ E. B. Karlsson and J. Mayers, Phys. Rev. Lett. 92, 249601 (2004).

${ }^{43}$ C. Stock, R. A. Cowley, J. W. Taylor, and S. M. Bennington, Phys. Rev. B 81, 024303 (2010).

${ }^{44}$ J. Mayers, N. I. Gidopoulos, M. A. Adams, G. Reiter, C. Andreani, and R. Senesi, Phys. Rev. B 84, 056301 (2011). 